\title{
Ectopic E2F expression induces $S$ phase and apoptosis in Drosophila imaginal discs
}

\author{
Maki Asano, Joseph R. Nevins, and Robin P. Wharton \\ Department of Genetics, Howard Hughes Medical Institute, Duke University Medical Center, Durham, North Carolina \\ 27710 USA
}

\begin{abstract}
Previous experiments suggest that a key event in the commitment of cultured mammalian cells to entering $S$ phase is a rise in activity of the transcription factor E2F. In this report, we study the role of Drosophila E2F in imaginal disc cells in vivo, by examining the distribution of the endogenous protein and studying the consequences of ectopic E2F expression. First, we find that endogenous E2F falls from high to very low levels as cells initiate DNA synthesis during a developmentally regulated $G_{1}-S$-transition in the eye disc. Second, we find that ectopic E2F expression drives many otherwise quiescent cells to enter $S$ phase. Subsequently, cells throughout the discs express reaper (a regulator of apoptosis) and then die. Third, we find that ectopic E2F expression during $S$ phase in normally cycling cells blocks their re-entry into $S$ phase in the following cell cycle. Although we do not know the fate of these cells, we suspect that ultimately they are killed by ectopic E2F. Taken together, our results show that an elevation in the level of E2F is sufficient to induce imaginal disc cells to enter $S$ phase. Furthermore, they suggest that the downregulation of E2F upon entry into $S$ phase may be essential to prevent the induction of apoptosis.
\end{abstract}

[Key Words: E2F; cell cycle; apoptosis; Drosophila; development]

Received February 29, 1996; accepted in revised version April 29, 1996

Recent work has shown that entry into $S$ phase is regulated by a complex pathway that culminates in the activation of the E2F transcription factors (Fig. 1). One of the initial events in this cascade is a rise in the level of the $\mathrm{G}_{1}$ cyclins that bind to and activate the cyclin-dependent kinases (CDKs) (Sherr 1993). These, in turn, phosphorylate and thereby inactivate members of the retinoblastoma $(\mathrm{Rb})$ family of "tumor suppressor" proteins (Weinberg 1995). In their unphosphorylated state, $\mathrm{Rb}$ proteins bind to members of the E2F family of DNA-binding proteins, preventing them from activating transcription. Phosphorylation of $\mathrm{Rb}$ leads to the accumulation of free, active E2F that stimulates the transcription of two classes of genes (for review, see Nevins 1992; Helin and Harlow 1993; La Thangue 1994). One class encodes proteins required for DNA synthesis, such as dihydrofolate reductase (DHFR), ribonucleotide reductase (RNR), proliferating cell nuclear antigen (PCNA), and DNA pola. The other class encodes cell-cycle regulators, including cyclin $\mathrm{E}$, cyclin $\mathrm{A}$, and several of the E2F genes themselves.

The idea that E2F activation commits cells to enter S phase derives from several lines of evidence. First, transcriptionally active E2F (i.e., E2F free of an inhibitory $\mathrm{Rb}$ family member) accumulates at the end of $G_{1}$, coincident with target gene activation (for review, see Nevins 1992). Second, E2F overexpression induces $S$ phase in quiescent cultured cells arrested as a consequence of serum starvation, $\gamma$-irradiation, or expression of CDK inhibitors (Johnson et al. 1993; Qin et al. 1994; Shan and Lee 1994; DeGregori et al. 1995b). Third, several DNA tumor viruses encode an early gene product that induces quiescent cells to enter $\mathrm{S}$ phase by inactivating $\mathrm{Rb}$ proteins and thereby activating E2F (for review, see Nevins 1994).

In the experiments described above, deregulation of E2F activity does not induce quiescent mammalian cells to resume normal cycling. Instead, the cells undergo p53dependent apoptosis after initially having entered $S$ phase (Qin et al. 1994; Shan and Lee 1994; Wu and Levine 1994; Kowalik et al. 1995). The DNA tumor viruses circumvent this E2F-induced cell death via viral proteins that inactivate p53 (Stein et al. 1990; Lowe and Ruley 1993; Sabbatini et al. 1995). Nevertheless, the activation of endogenous E2F during $G_{1}$ in a normal cell cycle does not lead to apoptosis, and it will be important to understand how E2F deregulation causes apoptosis.

Although much is known about E2F activity in vitro and in cultured cells, very little is known of its role in vivo. No mammalian E2F mutant has been reported. Moreover, it is likely that the various mammalian E2F proteins act redundantly (at least in part), complicating genetic analysis. In contrast, only a single E2F gene has been reported to date in Drosophila (Dynlacht et al. 


\section{Growth Stimulation}

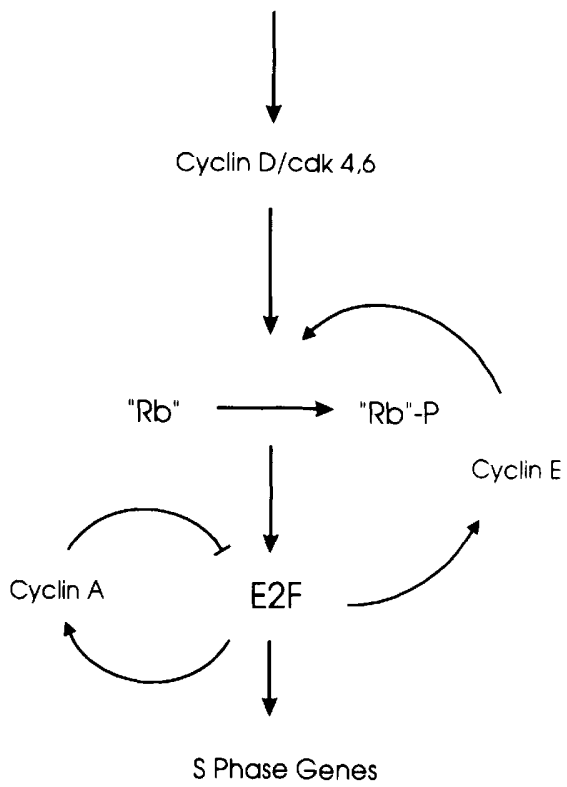

Figure 1. The scheme describes events during $G_{1}$ that lead to the inactivation of $\mathrm{Rb}$ and the accumulation of E2F, committing the cell to enter $\mathrm{S}$ phase. For references, see recent reviews by Weinberg (1995), Sherr (1993), and Nevins (1992).

1994; Ohtani and Nevins 1994). Many of the properties of Drosophila and mammalian E2F are similar; in particular, Drosophila E2F binds to DNA as a heterodimer with DP1 to activate transcription (Dynlacht et al. 1994; Ohtani and Nevins 1994). Duronio et al. (1995) recently have shown that Drosophila E2F is necessary for S phase in vivo; DNA synthesis does not occur in nuclear cycle 17 in neuroblasts or endocycling gut cells of E2F mutant embryos. However, these cells have unusual cycles, lacking $G_{1}$ and $G_{2}$ phases in one case, and $M$ phase in the other, and therefore, the role of E2F in Drosophila cells with canonical, four-phase cell cycles has not been tested. Moreover, it has been unclear whether the level of E2F normally limits entry into $S$ phase.

We have investigated the role of E2F in Drosophila imaginal disc cells which have four-phase cell cycles similar to those in mammalian cells. We find that in specially regulated cells of the eye imaginal disc E2F accumulates to a high level during $G_{1}$; the level of protein falls dramatically upon entry into $S$ phase. We also find that ectopic E2F expression is sufficient to commit many of the otherwise quiescent cells in the imaginal discs to enter $\mathrm{S}$ phase. Deregulation of E2F causes cells to express reaper, a specific effector of apoptosis (White et al. 1994), and then die. Finally, we observe that ectopic E2F expression in normally cycling cells prevents their re-entry into $\mathrm{S}$ phase in the following cycle. We infer that at least some of these cells are killed as a result of inappropriate E2F activity in $S$ phase. From these results we suggest that elevation of E2F during $G_{1}$ is sufficient to induce $S$ phase, and that normal progression through the cell cycle requires low levels of E2F after DNA synthesis has begun.

\section{Results}

Endogenous E2F accumulation during $a$ developmentally regulated $G_{1}-S$ transition in the eye imaginal disc

The imaginal discs are flattened sac-like invaginations of the larval epithelium. Each surface of the sac is distinct: The columnar cells of one surface (loosely referred to as the disc) give rise to most of the adult ectoderm, while the squamous cells of the peripodial membrane contribute less to adult structures (for review, see Cohen 1993). Except in the eye disc, quiescent, differentiating cells and proliferating cells are intermingled and not readily distinguishable. However, cells in the eye disc remain undifferentiated until the third larval instar when a wave front marked by an indentation named the morphogenetic furrow sweeps across the disc from posterior to anterior. Ahead of the furrow undifferentiated cells cycle asynchronously. As the furrow approaches, cells arrest in a prolonged $G_{1}$ phase that is maintained as they first enter and then exit the furrow. At this point, some of the cells differentiate into photoreceptor precursors and the others synchronously enter one additional $\mathrm{S}$ phase before they too differentiate (for review, see Wolff and Ready 1993).

We first examined the distribution of endogenous E2F in wild-type eye discs during the developmentally regulated $G_{1}-S$ transition described above. As shown in Figure 2A, high levels of E2F accumulate in the nuclei of the $\mathrm{G}_{1}$-arrested cells both within and flanking the furrow. Shortly thereafter (e.g., in cells immediately to the posterior), E2F levels plummet (Fig. $2 \mathrm{H}$ ), around the time these cells enter $\mathrm{S}$ phase.

Several lines of evidence show that the antibody we have used specifically recognizes E2F. First, there is a marked reduction in the level of E2F in heterozygous $E 2 F^{7271} /+$ mutant discs both in the furrow and elsewhere (Fig. 2C). Second, E2F protein is undetectable in homozygous E2F mutant embryos, whereas it accumulates to high levels in wild-type sibling embryos (Fig. $2 \mathrm{E}, \mathrm{F} \mid$. Third, when used in a Western blot, the antibody recognizes primarily a single protein (see below and Fig. 2D).

To define more precisely the temporal relationship between the decline in E2F levels and entry into $S$ phase, we performed the following double-labeling experiment. Wild-type discs were incubated briefly in a solution containing BrdU, and standard immunohistochemical methods were used to analyze the distributions of E2F and incorporated BrdU. As shown in Figure 2, G and I, cells bearing high levels of E2F and BrdU form abutting stripes, without extensive intermingling. Moreover, we do not see doubly labeled cells using a variety of detection methods (data not shown). Thus, during this developmentally regulated $G_{1}-S$ transition, entry into $S$ phase coincides temporally with a precipitous drop in E2F from maximal to minimal levels.

We also find that cells bearing moderate levels of E2F are distributed randomly throughout imaginal tissues that contain asynchronously cycling cells, consistent 
with the idea that E2F levels rise during $G_{1}$ and fall dramatically upon entry into $S$ phase.

Ectopic expression of E2F is lethal

To examine the consequences of ectopic E2F expression, we generated germline transformants bearing a fusion gene in which transcription of a Drosophila E2F cDNA is directed by the thermoinducible hsp70 promoter. Transformants were obtained at a normal frequency and exhibited no detectable growth disadvantage with respect to untransformed siblings when reared at the noninducing temperature. These observations suggest that the basal level of expression in the recovered lines is insignificant.

Larvae bearing the hsp70-E2F transgene do not survive a $30-$ min heat shock at $37^{\circ} \mathrm{C}$. In contrast, the survival of otherwise isogenic control larvae is unaffected by an identical heat shock. Administration of a single heat shock throughout most of larval or pupal development kills most animals bearing either two or four copies of the hsp70-E2F transgene. Several independently derived transformants exhibit similar properties. Therefore, in the experiments described below, we have used one line which bears four copies of the hsp70-E2F transgene.
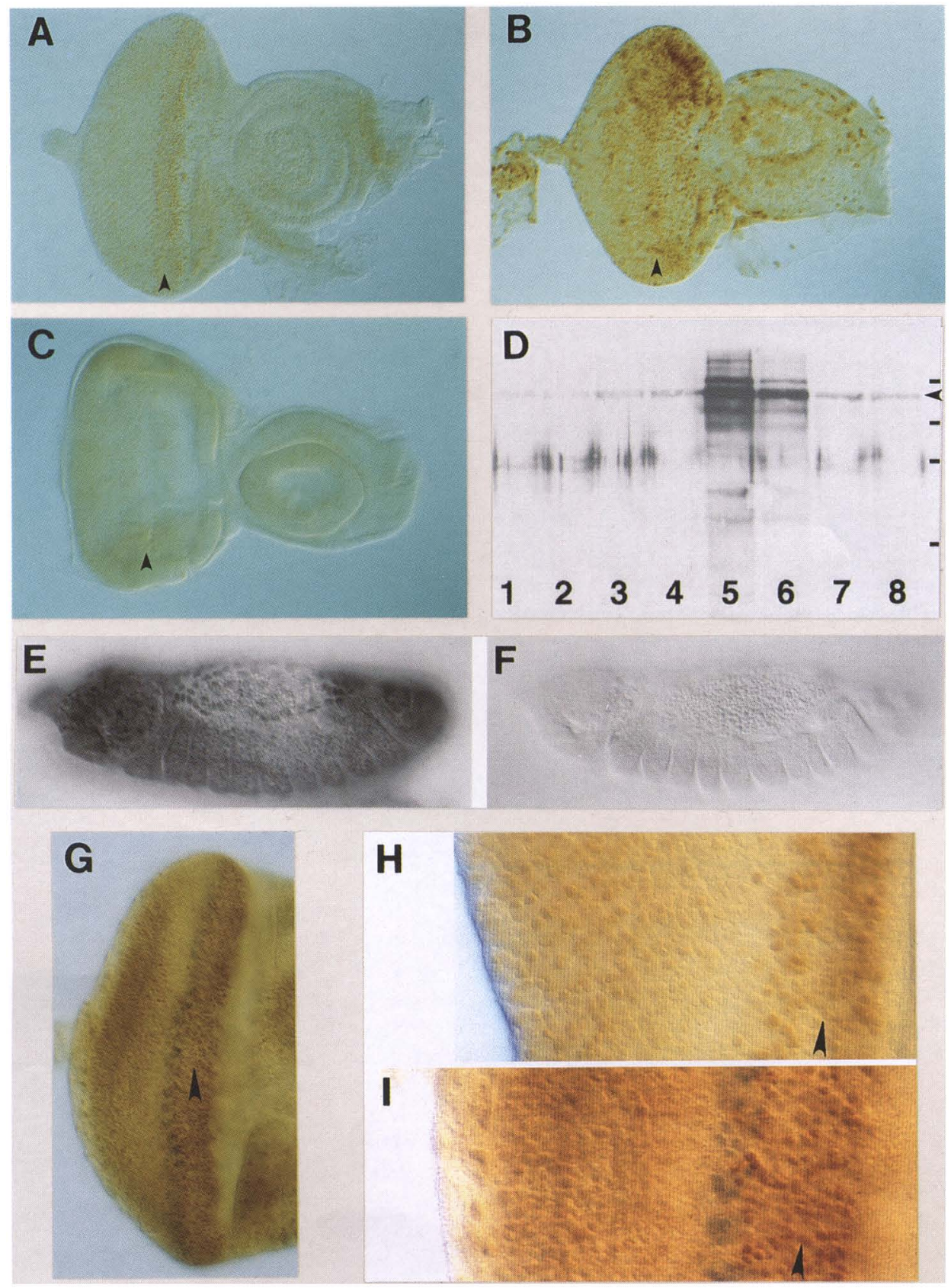

Figure 2. (See facing page for legend.) 
We next examined the distribution of E2F in imaginal discs following induction of the hsp70-E2F transgene. As shown in Figure 2B, eye discs from heat shocked hsp70-E2F larvae contain elevated levels of E2F throughout both the discs and the peripodial membranes. Following heat shock, excess E2F is detectable after $30 \mathrm{~min}$, accumulates to maximal levels after $\sim 1.5 \mathrm{hr}$, and is no longer apparent after $4 \mathrm{hr}$ (not shown; see Figure 2D).

To estimate the amount of excess E2F per disc following heat shock, we dissected eye-antennal discs from wild type and hsp70-E2F animals before and after heat shock, prepared protein extracts from these discs, and analyzed the level of E2F in these extracts on a Western blot. As shown in Figure 2D, the level of E2F in hsp70E2F discs rises substantially $1.5 \mathrm{hr}$ after heat shock and falls to control values by $8 \mathrm{hr}$ after heat shock. In contrast, the level of E2F in control discs is unaffected by heat shock. Serial dilution of the $1.5 \mathrm{hr}$ sample (not shown) suggests that total E2F protein levels rise $\sim 30$ fold during the experiment. However, the distribution of E2F is not uniform throughout the disc following induction, and thus, it is difficult to assess the level of excess E2F per cell. Nevertheless, based on the data in Figure 2, we estimate that the level of endogenous E2F falls $\geqslant 10$ fold as cells in the morphogenetic furrow exit $G_{1}$ and enter $S$ phase; and that in most cells, the excess E2F following heat shock is $\sim 10$-fold above the endogenous level.

\section{Ectopic E2F induces $S$ phase}

According to the regulatory scheme outlined in Figure 1, ectopic E2F expression should be sufficient to bypass the requirement for upstream signals (i.e., activation of CDKs) and drive cells into $S$ phase. To determine whether this is the case in vivo, discs from both control and hsp70-E2F larvae were incubated briefly in a solution containing BrdU, and the distribution of BrdU incorporated into DNA was detected by standard techniques.
The pattern of cells in S phase is essentially indistinguishable between wild-type eye discs and hsp70-E2F discs prepared from noninduced animals. BrdU-incorporating cells are scattered randomly throughout the eye disc anterior to the furrow, the attached antennal disc, and the peripodial membranes (Fig. 3A). In addition, a band of cells in the posterior of the eye disc synchronously enters $\mathrm{S}$ phase shortly after passage of the furrow. Essentially no other cells posterior to the furrow incorporate BrdU at this stage of development.

Ectopic E2F expression results in a marked increase in the number of $S$ phase cells in eye discs, as assayed by incorporation of BrdU (Fig. 3B). In particular, many cells posterior to the furrow enter $\mathrm{S}$ phase (Fig. 3B,C), whereas the corresponding cells in heat-shocked wild-type discs essentially never do so (Fig. 3A). Significantly, cells within and immediately flanking the furrow do not enter $S$ phase in response to ectopic E2F (Fig. 3C). Evidently, the $G_{1}$ arrest imposed by developmental signals associated with the morphogenetic furrow prevents these cells from responding to elevated levels of E2F. We also see ectopic S-phase induction throughout other imaginal tissues (not shown). Finally, we note that, whereas E2F levels rise almost immediately after heat shock, the induction of $\mathrm{S}$ phase lags behind, with the peak response occuring approximately $4 \mathrm{hr}$ after heat shock.

After passage of the morphogenetic furrow, developmentally uncommitted cells are sequentially recruited into clusters that comprise ommatidial precursors (Ready et al. 1976; Tomlinson and Ready 1987a,b). The nuclei in uncommitted cells occupy basal positions. As each photoreceptor precursor is recruited into a cluster, its nucleus simultaneously rises to an apical position (Tomlinson 1985), and it expresses the pan-neural antigen recognized by the $22 \mathrm{Cl0}$ monoclonal antibody (Fujita et al. 1982; Fig. 3F).

To identify cells posterior to the morphogenetic furrow that are driven into $S$ phase by ectopic E2F, we performed the following double-labeling experiment. Discs from heat-shocked hsp $70-E 2 F$ larvae were incubated

Figure 2. E2F accumulates in the $\mathrm{G}_{1}$-arrested cells of the morphogenetic furrow. (Top) The distribution of E2F in eye-antennal discs from wild-type $(A)$ and hsp70-E2F $(B)$ larvae $1.5 \mathrm{hr}$ post-heat shock. $(C)$ The distribution of E2F in a heterozygous $E 2 F^{7172}$ mutant disc (Duronio et al. 1995). In these and all subsequent photographs, the posterior of the eye disc is to the left and the attached antennal disc is to the right. The position of the morphogenetic furrow is indicated with an arrowhead in each case. Note that much of the E2F visible in $B$ is in nuclei of the overlying peripodial membrane (particularly the large nuclei evident in the photograph). Also note that the pattern of E2F accumulation is similar in wild-type and $E 2 F^{7172} /+$ discs: The highest level is seen in cells of the morphogenetic furrow in each case. Discs were fixed and stained together, each genotype marked by differentially dissecting the attached larval carcasses. $(D)$ A Western blot detecting the E2F in extracts of eye-antennal discs prepared from various larvae as follows: $E 2 F^{7172} /+$ (lane 1); untreated $w^{1118}$ and hsp70-E2F (lanes 2,3, respectively); $w^{1118} 1.5 \mathrm{hr}$ after heat shock (lane 4); hsp70-E2F 1.5 hr (lane 5), $4 \mathrm{hr}$ (lane 6), $8 \mathrm{hr}$ (lane 7), and $10 \mathrm{hr}$ (lane 8) after heat shock. The arrowhead marks the major E2F species, which has an apparent molecular weight substantially greater than that calculated from the sequence of the gene $(87.5 \mathrm{kD})$. The dashes at right indicate the mobility of molecular mass standards as follows (top to bottom): $116,97.4,66$, and $45 \mathrm{kD}$. Note that the irregular smear near the $66 \mathrm{kD}$ marker is an irreproducible artifact. $(E, F)$ The specificity of the anti-E2F antibodies used in these experiments. Shown are dorsolateral views of stage 13 sibling embryos from a $E 2 F^{7172} /+$ stock. E2F is readily detectable in the cells of the amnioserosa and the epidermis in the wild-type embryo in $E$, but no specific staining is evident in the presumptive $E 2 F^{7172} / E 2 F^{7172}$ homozygote in $F$. Approximately one-quarter of the stage 13 embryos from $E 2 F^{7172} /$ + flies are similar to the embryo shown in $F$, whereas all the stage 13 embryos from wild-type flies are similar to the embryo in $E$. $(G-I)$ E2F levels fall as $G_{1}$-arrested cells leave the furrow and enter $S$ phase. $(H)$ High magnification view of the distribution of endogenous E2F in a wild-type eye disc. Note that the level of E2F apparently falls precipitously in cells recently emerged from the furrow as they enter $\mathrm{S}$ phase $(G, I) .(G, I)$ Low and high magnification views, respectively, of the distributions of endogenous E2F (red-brown) and incorporated BrdU (blue-gray) in a wild-type eye disc. 


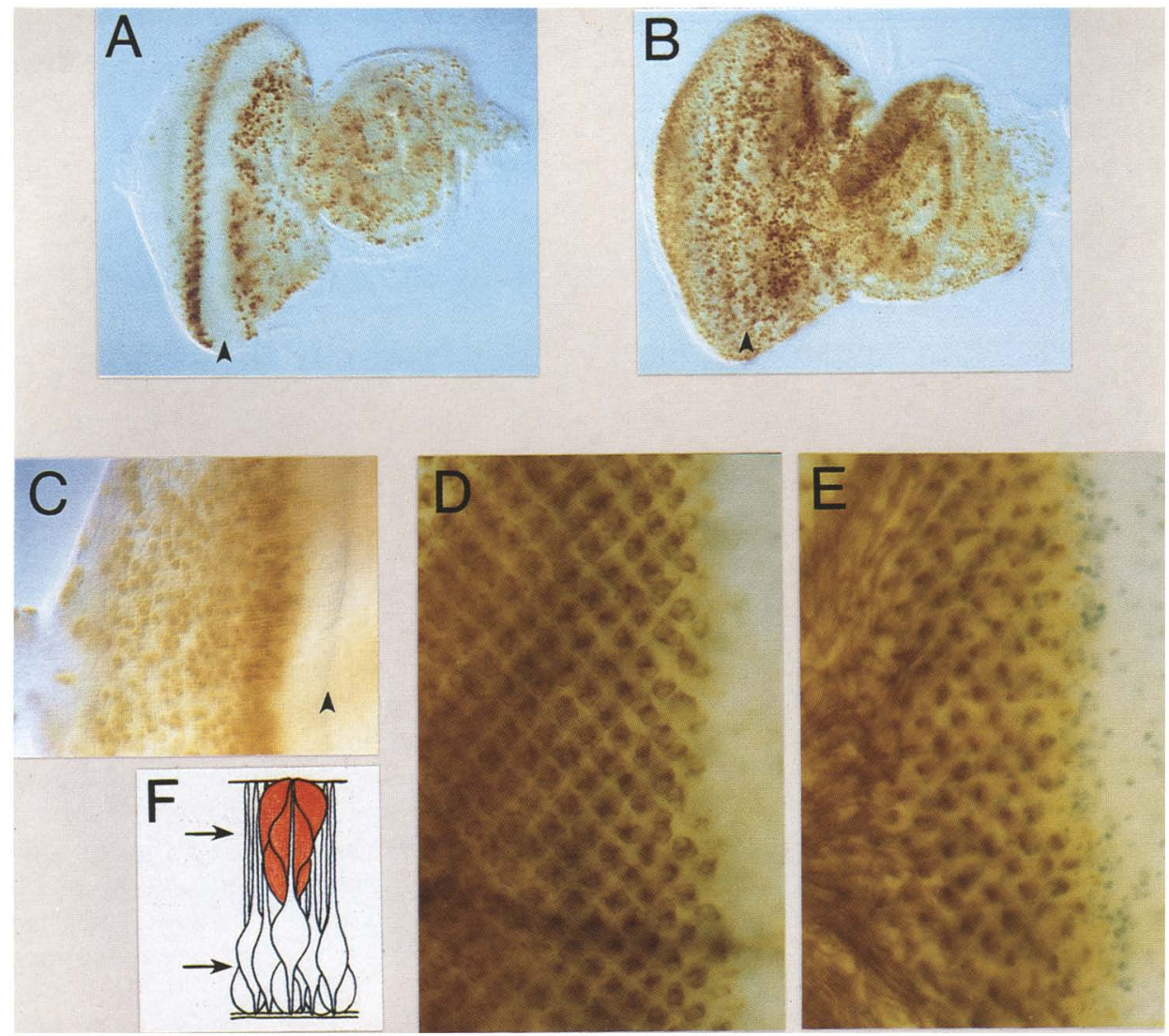

Figure 3. Induction of $\mathrm{S}$ phase by ectopic E2F. Shown at top are the distributions of incorporated BrdU in eye-antennal discs from wild-type $(A)$ and hsp70-E2F $(B)$ larvae $4 \mathrm{hr}$ post-heat shock. Cells in the peripodial membrane and cells in the imaginal disc are not readily distinguishable in these preparations. $(C)$ A high magnification view of incorporated BrdU in a heat-shocked hsp70-E2F eye disc from which the peripodial membrane has been dissected away. This preparation reveals that cells in $S$ phase are distributed throughout the region posterior to the furrow (arrowhead), but such cells are not seen immediately anterior to or within the furrow. $(D, E)$ High magnification apical and basal views, respectively, of the distributions of incorporated BrdU (blue-gray) and the $22 \mathrm{Cl0}$ antigen expressed by photoreceptor precursors (brown) in the posterior region of a hsp70-E2F eye disc 4 hr post-heat shock. The arrows in the schematic drawing in $F$ (adapted from a drawing by Wolff and Ready 1993) indicate the approximate focal planes in $D$ (top) and $E$ (bottom); the four red cells in this drawing represent 22C10-positive cells within a single ommatidial precluster, each with an apical nucleus. Note that the morphogenetic furrow is at the right margin of the photographs in $D$ and $E$. Also note that essentially no blue BrdU-containing nuclei are seen apically $(D)$, whereas such nuclei are present basally $(E)$ underneath the nuclei of the $22 \mathrm{Cl0}$-positive cells.

briefly in a solution containing BrdU, and then assayed for incorporated $\mathrm{BrdU}$ and the $22 \mathrm{C} 10$ antigen.

Figure 3, D and E, shows apical and basal views, respectively, of a representative preparation. Neither the photoreceptor cells (which express the $22 \mathrm{C} 10$ antigen) nor any of the other differentiated cells that also have apical nuclei enter $S$ phase in response to ectopic E2F. Instead, the nuclei driven into $\mathrm{S}$ phase occupy basal positions, in the same plane as nuclei in the synchronous band posterior to the furrow that is also present in wildtype discs. Thus, ectopic E2F apparently induces $S$ phase primarily in developmentally uncommitted cells and not in terminally differentiated cells that have been recruited into ommatidial precursors.

Three additional observations are consistent with the idea that terminally differentiated cells in the eye disc are relatively resistant to the effects of ectopic E2F. First, recruitment of photoreceptors to ommatidial clusters (assayed by the distribution of $22 \mathrm{C} 10$ antigen) is essentially unperturbed by ectopic E2F (Fig. 3D,E). Second, the cone cells, which are recruited into clusters following the recruitment of all 8 photoreceptors, tolerate very high levels of E2F following heat shock without entering $S$ phase (Fig. 3D,4A). Third, ectopic E2F has a relatively minor effect on the organization of the mature eyes of adults that develop from the rare larvae that survive heat shock (Fig. 4C). Thus, in the posterior region of the eye disc, ectopic E2F appears to induce developmentally uncommitted cells, but not terminally differentiated ones, to enter $\mathrm{S}$ phase.

\section{Ectopic E2F induces apoptosis}

The ectopic expression of $\mathrm{E} 2 \mathrm{~F} 1$ in $\mathrm{G}_{0}$-arrested mammalian cells induces entry into $S$ phase, which is followed by apoptosis (Qin et al. 1994; Shan and Lee 1994; Wu and 


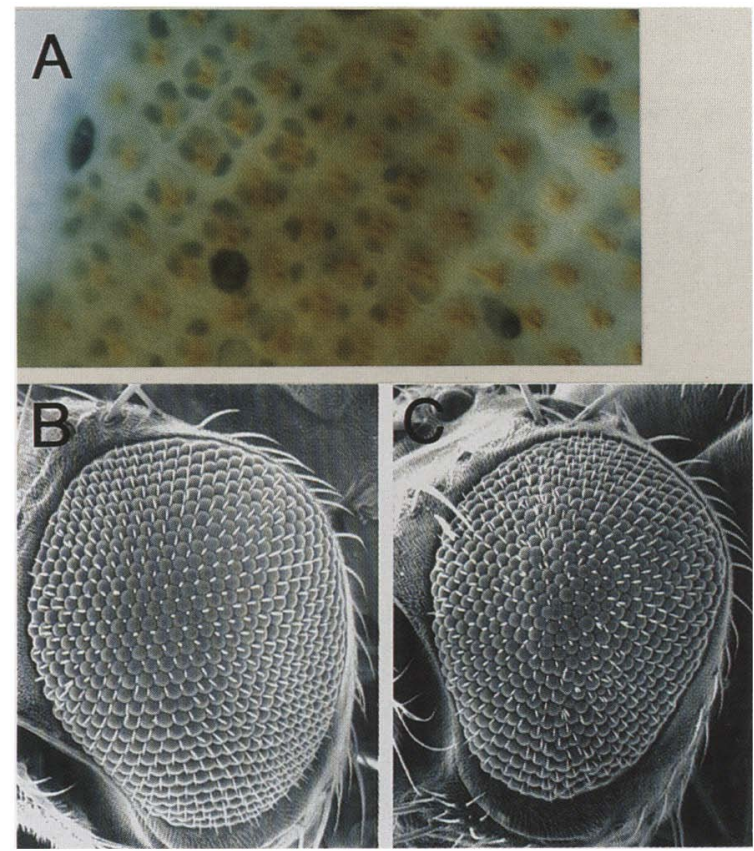

Figure 4. Terminally differentiated cells are resistant to ectopic E2F. Shown at top $(A)$ are the distributions of E2F (bluegray) and the $22 \mathrm{Cl} 0$ antigen (brown) expressed by photoreceptor precursors at the posterior margin of a hsp $70-\mathrm{E} 2 \mathrm{~F}$ eye disc $1.5 \mathrm{hr}$ post-heat shock. In this region of the disc, E2F accumulates to particularly high levels in the apically positioned cone cell nuclei, which are recruited later to ommatidial clusters and lie above the photoreceptor nuclei (out of the focal planel. Nevertheless, these and other terminally differentiated cells do not enter S phase in response to ectopic E2F (Fig. 3), and only minor defects are evident in the eyes of rare adults that develop from heat-shocked larvae, as shown at the bottom. Scanning electron micrographs of adult eyes from heat-shocked wild-type $(B)$ and hsp70-E2F $(C)$ larvae are shown at bottom. In the latter case, note the presence of irregular ommatidial rows, fused ommatidia, and duplicated interommatidial bristles.

Levine 1994; Kowalik et al. 1995). However, it has not been clear whether this E2F-mediated apoptosis reflects an intrinsic regulatory capacity of E2F. In particular, it is possible that the treatments used to induce cell cycle arrest (e.g., serum withdrawal, $\gamma$ irradiation) either deprive the cells of other signals essential for normal cell cycle progression or impose blockades downstream of the $\mathrm{G}_{1}-\mathrm{S}$ transition. Accordingly, we wished to determine whether ectopic expression of E2F in vivo also induces apoptosis in the absence of other treatments that might interfere with cell cycle progression.

To monitor cell death, we prepared imaginal discs from either wild-type or hsp70-E2F larvae at various times after heat shock, incubated them briefly in a solution containing the vital dye acridine orange, and immediately examined them for the presence of fluorescent, dying cells. We find that ectopic expression of E2F causes extensive cell death in all of the imaginal discs, including the eye-antennal disc (Fig. 5B). In contrast, very little cell death is apparent in control discs from either hsp70-
E2F larvae not subjected to heat shock or from heatshocked wild-type larvae (Fig. 5A). The number of dying cells peaks $\sim 8-10 \mathrm{hr}$ after induction of the hsp70-E2F transgene. Thus, cell death lags $\sim 4-6 \mathrm{hr}$ behind the peak of induced $\mathrm{S}$ phase, as described above.

In one region of the eye disc, the cells are resistant to ectopic E2F, neither entering $S$ phase nor undergoing apoptosis. As shown in Figure 5B, most of the hsp70-E2F eye discs bear a stripe containing no dying, fluorescent cells (Fig. 5B); no such fluorescence-free zone is consistently observed in wild-type eye discs (Fig. 5A) or in other discs from either wild-type or hsp70-E2F larvae (not shown). This stripe includes cells in and around the morphogenetic furrow, where developmental signals prevent cells from entering $S$ phase in response to ectopic E2F (Fig. 3C). Thus, within the eye disc, cells in and around the furrow do not enter $\mathrm{S}$ phase and do not die in response to ectopic E2F, whereas cells elsewhere first enter $\mathrm{S}$ phase and subsequently die. These observations are consistent with the idea that individual cells first enter S phase and then die in response to ectopic E2F.

We have also examined the expression of reaper $(r p r)$ to determine whether the cell death resulting from E2F misexpression is apoptotic. rpr encodes an effector of programmed cell death in Drosophila (White et al. 1994; Hay et al. 1995). As shown in Figure 6, B and D, ectopic E2F expression leads to the induction of rpr mRNA in cells throughout the eye and wing discs as well as in other discs (not shown). In contrast, control discs bear
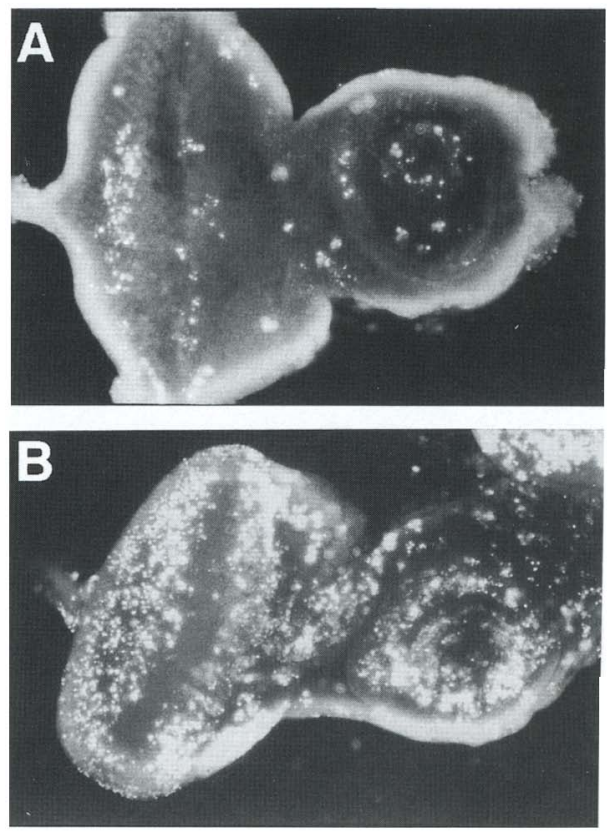

Figure 5. Induction of cell death by ectopic E2F. Shown are the distributions of dying cells in eye-antennal discs from wild-type $\langle A|$ and hsp70-E2F $\langle B|$ larvae, $8 \mathrm{hr}$ post-heat shock. Dying cells are visualized by incubation with acridine orange and fluorescence microscopy. The fluorescence-free zone that includes the morphogenetic furrow in hsp70-E2F discs is indicated with an arrow. 
Figure 6. Induction of reaper mRNA expression by ectopic E2F. Shown are the results of in situ hybridization experiments that reveal the distribution of reaper mRNA in discs from wild-type (left $)$ and hsp70-E2F (right) larvae $7 \mathrm{hr}$ post-heat shock. $(A, B)$ Eye-antennal discs photographed with Normarski optics; $(C, D)$ wing discs, anterior at the bottom, photographed with bright-field optics.
WT
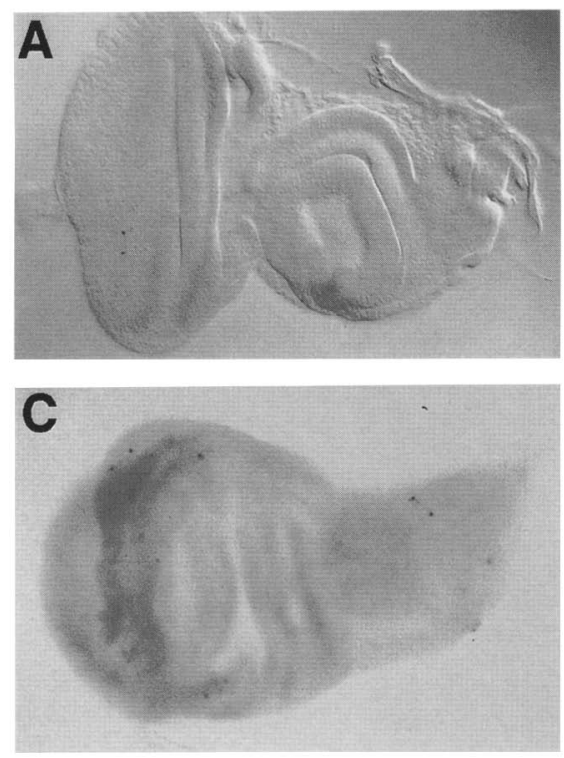

hsp-E2F
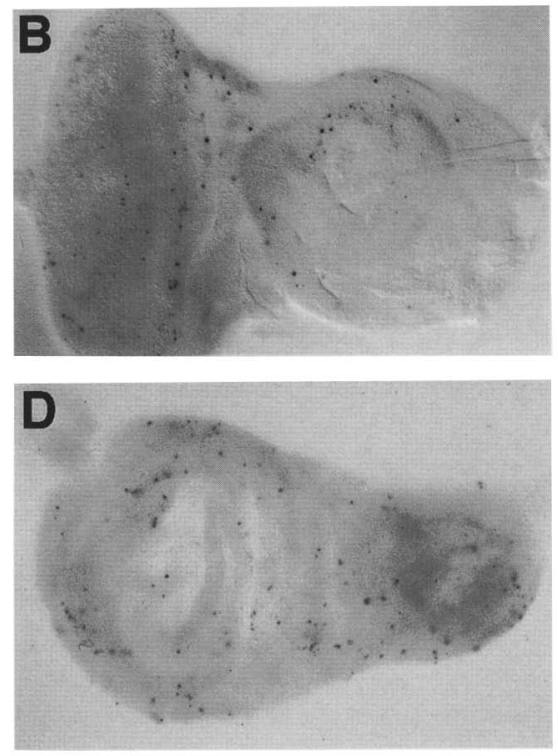

only a few cells expressing detectable rpr mRNA at this stage of development (Fig. 6A,C). As is the case for apoptotic cells in embryos (White et al. 1994), the rpr expression that we observe in discs precedes by $1-2 \mathrm{hr}$ failure of acridine orange exclusion. Finally, we note that the number of cells expressing rpr at any point apparently is smaller than the number of cells that later stain with acridine orange. In part, this may be attributable to the time course of rpr mRNA expression, which is detectable only transiently (data not shown). Another possibility, which is supported by a recent report (Grether et al. 1995), is that rpr is only one of several partially redundant cell death effectors.

\section{Ectopic E2F affects cycling cells}

The results described above can be accounted for by the ability of ectopic E2F to drive quiescent cells into $S$ phase (Fig. 3). However, the observations described below suggest that ectopic E2F has a profound effect on essentially all of the asynchronously cycling cells in the imaginal discs. In particular, we infer that cells in $S$ phase are sensitive to ectopic E2F.

As described above and in Figure 3, ectopic E2F expression drives cells to enter $\mathrm{S}$ phase $4 \mathrm{hr}$ after heat shock. However $10 \mathrm{hr}$ after heat shock, essentially none of the imaginal disc cells from hsp70-E2F larvae is in S phase (Fig. 7B,D). With the exception of a narrow band of cells in the eye disc, no BrdU incorporation is detectable in cells in the eye disc, the attached antennal disc, the wing disc (or other discs), or the peripodial membranes. In contrast, cells in $S$ phase are distributed throughout the discs from wild-type larvae $10 \mathrm{hr}$ post-heat shock (Fig. 7A,C). We interpret these observations as follows: One cell cycle in imaginal discs is $8.5-11.9 \mathrm{hr}$ long (Postelthwait 1978). Thus, some wild-type cells in S phase at the time of heat shock reenter $S$ phase on schedule $10 \mathrm{hr}$ later, whereas cells from hsp70-E2F discs in $S$ phase at the time of heat shock do not. Apparently, ectopic E2F accumulation during $S$ phase prevents normal progression into the following cell cycle. Although we do not know the fate of these cells, we suspect they are killed by ectopic E2F and contribute to the cell death described in Figures 5 and 6.

Based on their position in the disc relative to the morphogenetic furrow, we believe that the narrow band of $S$ phase cells in hsp70-E2F eye discs (Fig. 7B) was within the furrow at the time of heat shock. As described above, developmental signals in the furrow prevent cells from entering S phase (Fig. 3) and dying (Fig. 5) in response to ectopic E2F. Ten hours later, having emerged from the furrow, some of these cells synchronously enter $S$ phase on schedule.

Finally we note that ectopic E2F does not cause a global inhibition of DNA synthesis. Ten hours after heat shock, the pattern of BrdU incorporation in larval brains from either wild-type or hsp70-E2F animals is essentially indistinguishable (Fig. 7E,F).

\section{Discussion}

The observations presented here suggest that modulation of the level of E2F during the $G_{1}-S$ transition is essential in vivo. During a developmentally regulated $\mathrm{G}_{1}-\mathrm{S}$ transition in the eye imaginal disc, we find that $\mathrm{E} 2 \mathrm{~F}$ protein accumulates to high levels during $G_{1}$ and then falls $\sim 10$ - to 20 -fold as the cells initiate DNA synthesis. The level of E2F in $G_{1}$ may limit entry into $S$ phase. In support of this idea, we find that ectopic E2F drives $G_{1}$ arrested cells, and perhaps others, into $S$ phase. Furthermore, downregulation of E2F protein upon entry into $S$ phase may also be essential, as we find that ectopic E2F 
WT
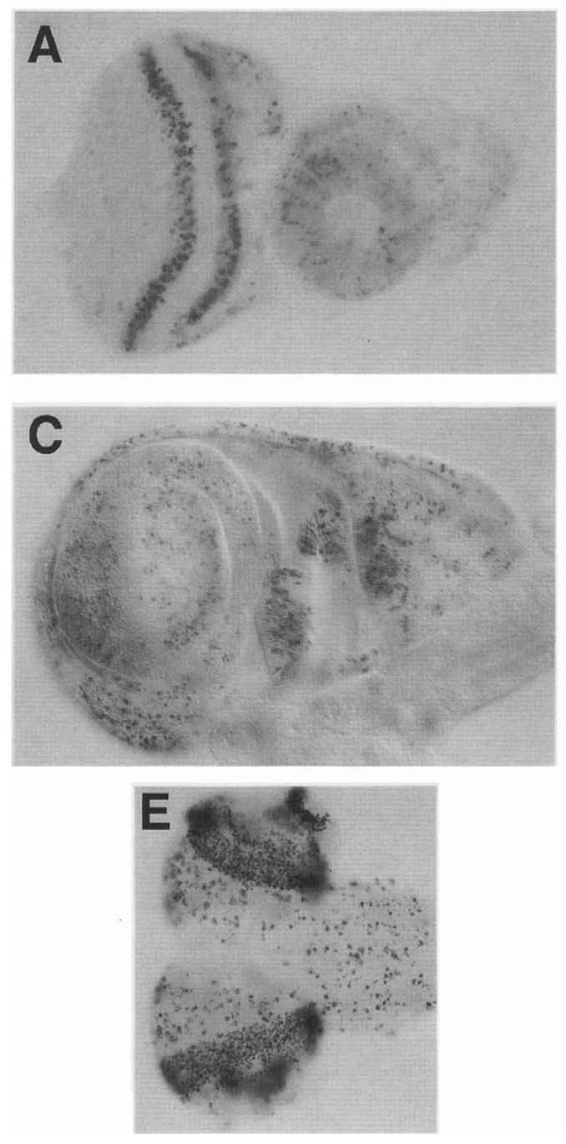

hsp-E2F
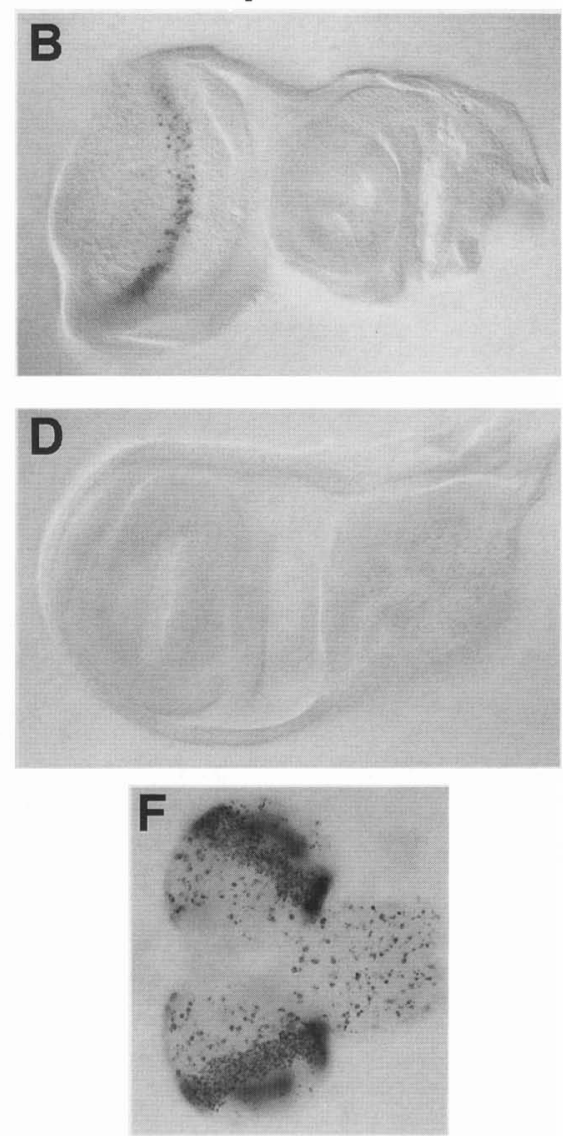

Figure 7. Effect of ectopic E2F on cycling cells. Shown are the distributions of incroporated BrdU in tissue from wild-type (left) and hsp70-E2F (right) larvae $10 \mathrm{hr}$ postheat shock. $(A, B)$ eye-antennal discs; $(C, D)$ wing discs (anterior at the bottom); $(E, F)$ portion of the CNS. The position of the morphogenetic furrow in $A$ and $B$ is indicated with an arrowhead. Note that a piece of extraneous tissue occludes the anterior margin of the wing disc in $C$, and that part of the optic lobe in $E$ is slightly torn. It is difficult to examine BrdU incorporation at later time points, because of larval lethality and fragility of the discs. In addition, the morphology of discs from hsp70-E2F larvae $10 \mathrm{hr}$ post-heat shock is disrupted, presumably as a consequence of extensive cell death. expression during $S$ phase prevents cycling cells from subsequently reentering $S$ phase on schedule.

\section{Elevated E2F activity during $G_{1}$ commits cells to enter $S$ phase}

Our experiments demonstrate that ectopic accumulation of E2F is sufficient to induce $S$ phase in the normally quiescent cells posterior to the morphogenetic furrow, many of which are arrested in $G_{1}$. This view is consistent with the observation that E2F is required for S phase in the Drosophila embryo (Duronio et al. 1995) as well as with previous observations that E2F activity accumulates during the $G_{1}$ phase of mammalian cell cycles (Nevins 1992). Although we cannot be certain that accumulation of endogenous E2F during $G_{1}$ performs the same role, the accumulation of E2F to high levels in cells held in $G_{1}$ by developmental signals is consistent with this idea. Taken together, these observations provide strong evidence that the timely accumulation of E2F during $G_{1}$ leads to the initiation of $S$ phase.

Despite evidence that ectopic E2F can drive some cells into $S$ phase, at least two cell types are resistant to the elevated E2F levels achieved in the experiments reported here. Cells in and around the morphogenetic furrow tolerate high levels of endogenous protein for an extended period without entering $\mathrm{S}$ phase, and they are not driven into $S$ phase by ectopic E2F expression. Apparently the $G_{1}$ arrest imposed on furrow cells by unknown developmental signals is sufficiently potent to resist E2F action.

Terminally differentiated cells that have been recruited into ommatidial clusters comprise a second class of cells that are resistant to ectopic E2F. We do not understand the mechanism by which differentiated cells in the eye disc tolerate levels of E2F that are well above the threshold capable of inducing $\mathrm{S}$ phase (e.g., the cone cells and their neighbors in Figs. 3 and 4).

\section{Down-regulation of E2F activity during $S$ phase may be essential}

The idea that E2F levels must be low during $\mathrm{S}$ phase to allow normal progression through the cell cycle is suggested by our observation that, in the synchronized cells associated with the furrow, the amount of E2F falls dramatically as these cells enter $\mathrm{S}$ phase. Moreover, from the analysis of BrdU incorporation in discs $10 \mathrm{hr}$ after misexpression of E2F, we conclude that this downregulation may be essential for normal cell cycle progression and cell survival. This conclusion is based on the following argument.

The cell cycle in imaginal disc cells lasts 8.5 to $11.9 \mathrm{hr}$ 
(Postlethwait 1978). Thus, any cell in S phase when the hsp70-E2F transgene is induced should return to $S$ phase approximately $10 \mathrm{hr}$ later, unless ectopic E2F influences its progression into the subsequent cell cycle. Because we find essentially no BrdU incorporation $10 \mathrm{hr}$ after heat shock, we infer that cells in $S$ phase are acutely sensitive to ectopic E2F. Cells in $G_{2}$ or $M$ phase are unmarked in our experiments, and thus we do not know whether they too are sensitive to ectopic E2F. Although we have not identified individual cells that are in S phase at the time of heat shock to determine their fate, we suspect they are killed by ectopic E2F, as are, for example, the normally quiescent cells posterior to the morphogenetic furrow.

\section{Roles for cyclin $E$ and E2F in regulating $S$ phase entry}

Based on the data presented here, we conclude that elevated levels of E2F are sufficient to commit imaginal disc cells to enter $S$ phase. As a corollary, we believe that the level of E2F normally limits commitment in vivo. However, recent studies have shown that ectopic expression of cyclin $\mathrm{E}$ under the control of the hsp70 promoter induces eye imaginal disc cells to enter $\mathrm{S}$ phase (Richardson et al. 1995). How can both cyclin E and E2F be limiting during $\mathrm{G}_{1}$ ?

Based on several observations, we believe it is likely that E2F and cyclin E collaborate to mediate the induction of $S$ phase. In the regulatory scheme of Figure 1, the relationship between E2F and cyclin $E$ is complex, with recent evidence suggesting that cyclin $\mathrm{E}$ acts both upstream of E2F by promoting the phosphorylation of $\mathrm{Rb}$ family members (Hinds et al. 1992) and downstream as a transcriptional target of E2F (DeGregori et al. 1995a; Duronio and O'Farrell 1995; Ohtani et al. 1995). Thus, E2F and cyclin E may act synergistically, each enhancing the accumulation of the other.

Ectopic expression of either cyclin E (Knoblich et al. 1994; Richardson et al. 1995) or E2F, as reported here, drives cells into $S$ phase. Given the regulatory relationships linking cyclin $\mathrm{E}$ and $\mathrm{E} 2 \mathrm{~F}$, it is possible that ectopic cyclin $\mathrm{E}$ acts by enhancing the accumulation of E2F activity or that it acts downstream and independently of E2F. Duronio and O'Farrell (1995) argued in favor of the latter explanation based on their observation that ectopic cyclin E could induce S phase in E2F-deficient embryos. However, ectopic cyclin E could not induce $S$ phase in slightly older embryos, raising the possibility that the cyclin E-mediated S phase they observe relies upon a residual supply of maternal E2F.

Other observations suggest that E2F and cyclin E may act primarily at different steps in $G_{1}$ during the preparation to enter $S$ phase. Ectopic cyclin $E$ induces $S$ phase within $60 \mathrm{~min}$ of heat shock (Knoblich et al. 1994; Richardson et al. 1995), whereas the E2F-mediated induction of $S$ phase is not maximal until $4 \mathrm{hr}$ after heat shock. In addition, among the synchronized cells of the furrow, cyclin $\mathrm{E}$ accumulates later than does E2F. Taken together, these differences suggest that cyclin $\mathrm{E}$ overproduction accelerates passage of cells through late $G_{1}$, pro- moting premature entry into an essentially normal $S$ phase. According to this view, ectopic cyclin E would not induce premature $S$ phase in the absence of E2F. In contrast, ectopic E2F induces an abnormal S phase that results in the death of the cell, as discussed below.

\section{E2F-induced apoptosis}

Our experiments show that ectopic E2F expression in Drosophila imaginal discs induces accumulation of $r p r$ mRNA and apoptosis. Yet, during a normal cell cycle, activation of E2F presumably occurs in every $G_{1}$ phase without lethal consequences. Why, then, does ectopic E2F activity induce apoptosis?

One explanation is that cells driven into $\mathrm{S}$ phase by ectopic E2F fail to receive additional signals that are essential for normal proliferation, leading to the generation of a "signal imbalance" (Fisher 1994; Harrington et al. 1994|. While the molecular mechanisms underlying this idea are unclear, one possibility is suggested by the action of DNA tumor viruses which simultaneously liberate endogenous E2F and inhibit p53 upon infection (Nevins 1992). Perhaps the signal imbalance is measured by p53. For example, E2F accumulation during $G_{1}$ might activate p53, either directly or indirectly, a condition which leads to apoptosis if left unchecked. During normal proliferation, however, the products of other pathways might inhibit the activated p53, much as occurs during DNA tumor virus infection.

An alternative explanation is that excess E2F activity during $S$ phase results in aberrant replication events, leading to the induction of apoptosis. This idea is consistent with our findings that E2F is down-regulated upon S-phase entry and that ectopic E2F accumulation during $S$ phase prevents cycling cells from subsequently reentering $S$ phase, presumably by killing them. Evaluation of these idea awaits elucidation of the pathway linking E2F-dependent transcription and the initiation of DNA synthesis.

\section{Materials and methods}

Plasmids and transgenic flies

DNA encoding the hsp70-E2F chimeric gene was constructed by inserting the $3.8 \cdot \mathrm{kb}$ EcoRI to the ApaI fragment of a Drosophila E2F1 cDNA clone (Ohtani and Nevins 1994) between DNA encoding the hsp70 promoter and the $\alpha$-tubulin 84B 3' UTR and polyadenylation signals in the P element transforming vector Casper. Transformants were generated by microinjection of $w^{1118}$ embryos by standard methods. E2F alleles were described by Duronio et al. (1995). Heat shocks were administrated in glass tubes in a $37^{\circ} \mathrm{C}$ water bath for $30 \mathrm{~min}$.

\section{Scanning electron microscopy}

Adult flies were fixed in ethyl alcohol by standard methods and critical point-dried. Flies were coated with gold-palladium (80:20). 


\section{Histology and immunochemistry}

Discs were dissected from late third instar wandering larvae in Ringer's solution, fixed in $2 \%$ paraformaldehyde (Polysciences) in PBS for 20 min at room temperature, washed in PBS, permeabilized in PBS containing $0.1 \%$ Saponin and $1 \%$ normal goat serum, and incubated with monoclonal antibody $22 \mathrm{C} 10$. For visualization of E2F, rabbit polyclonal antisera (Ohtani and Nevins 1994) were purified by ammonium sulfate fractionation and protein A-agarose affinity chromatography (Bio-Rad). For double-staining of $22 \mathrm{Cl} 0$ and E2F, discs were incubated simultaneously with both primary antibodies, and then sequentially with the appropriate secondary antibodies. $22 \mathrm{C} 10$ was visualized with $\mathrm{DAB}$, and E2F was visualized with the SG substrate (Vector). Visualization of E2F on Western blots was by standard methods using ECL detection reagents (Amersham).

\section{BrdU labeling}

Labeling and detection of cells in S phase was performed as described previously (Wilder and Perrimon 1995) with minor modifications. Discs were dissected in Ringer's solution, and then incubated in the presence of $50 \mu \mathrm{g} / \mathrm{ml} \mathrm{BrdU}$ (Sigma) for 30 min. Anti-BrdU antibody (Zymed) was used either alone or in combination with anti-E2F for double-labeling, as described above.

\section{Acridine orange staining}

Acridine orange staining was carried out as described (Spreij 1971) using acridine orange (Sigma) at $1.6 \times 10^{-7} \mathrm{M}$.

\section{Whole-mount in situ hybridization}

In situ hybridization using digoxigenin-labeled double-stranded rpr DNA (Genius kit, Boehringer Mannheim) was performed essentially as described by Tautz and Pfeifle (1989).

\section{Acknowledgments}

We thank T. Lee and M. Patterson for excellent technical help, R. Fehon for antibodies and advice, N. Dyson for strains, L. Eibest for performing the electron microscopy, K. Culler and S. Bowling for help preparing the manuscript, and J. DeGregori, B. Hay, J. Heitman, D. Lew, D. Morisato, K. Ohtani, A. Sancar, P. Spierer, and A. Tomlinson for helpful discussions or comments on the manuscript. This work was supported by the Howard Hughes Medical Institute.

The publication costs of this article were defrayed in part by payment of page charges. This article must therefore be hereby marked "advertisement" in accordance with 18 USC section 1734 solely to indicate this fact.

\section{References}

Cohen, S.M. 1993. Imaginal disc development. In The development of Drosophila melanogaster, Vol. II /ed. M. Bate and A. Martinez Arias), pp. 747-841, Cold Spring Harbor Laboratory Press, Cold Spring Harbor, NY.

DeGregori, J., T. Kowalik, and J.R. Nevins. 1995a. Cellular targets for activation by the E2Fl transcription factor include DNA synthesis and G1/S regulatory genes. Mol. Cell. Biol. 15: 4215-4224

DeGregori, J., G. Leone, K. Ohtani, A. Miron, and J.R. Nevins. 1995b. E2F1 accumulation bypasses a $G_{1}$ arrest resulting from the inhibition of $G_{1}$ cyclin-dependent kinase activity.
Genes \& Dev. 9: 2873-2887.

Duronio, R.J. and P.H. O'Farrell. 1995. Developmental control of the $\mathrm{G}_{1}$ to $\mathrm{S}$ transition in Drosophila: Cyclin $\mathrm{E}$ is a limiting downstream target of E2F. Genes \& Dev. 9: 1456-1468.

Duronio, R.J., P.H. O'Farrell, J.-E. Xie, A. Brook, and N. Dyson. 1995. The transcription factor E2F is required for $S$ phase during Drosophila embryogenesis. Genes \& Dev. 9: 14451455.

Dynlacht, B.D., A. Brook, M. Dembski, L. Yenush, and N. Dyson. 1994. DNA-binding and trans-activation properties of Drosophila E2F and DP proteins. Proc. Natl. Acad. Sci. 91: 6359-6363.

Fisher, D.E. 1994. Apoptosis in cancer therapy: Crossing the threshold. Cell 78: 539-542.

Fuiita, S.C., S.L. Zipursky, S. Benzer, A. Ferrus, and S.L. Shotwell. 1982. Monoclonal antibodies against the Drosophila nervous system. Proc. Natl. Acad. Sci. 79: 7929-7933.

Grether, M.E., J.M. Abrams, J. Agapite, K. White, and H. Steller. 1995. The head involution defective gene of Drosophila melanogaster functions in programmed cell death. Genes \& Dev. 9: 1694-1708.

Harrington, E.A., A. Fanidi, and G.I. Evan. 1994. Oncogenes and cell death. Curr. Opin. Genet. Dev. 4: 120-129.

Hay, B.A., D.A. Wassarman, and G.M. Rubin. 1995. Drosophila homologs of baculovirus inhibitor of apoptosis proteins function to block cell death. Cell 83: 1253-1262.

Helin, K. and E. Harlow. 1993. The retinoblastoma protein as a transcriptional repressor. Trends Cell Biol. 3: 43-46.

Hinds, P.W., S. Mittnacht, V. Dulic, A. Arnold, S.I. Reed, and R.A. Weinberg. 1992. Regulation of retinoblastoma protein functions by ectopic expression of human cyclins. Cell 70: 993-1006.

Johnson, D.G., J.K. Schwarz, W.D. Cress, and J.R. Nevins. 1993. Expression of transcription factor E2Fl induces quiescent cells to enter S phase. Nature 365: 349-352.

Johnson, D.G., K. Ohtani, and J.R. Nevins. 1994. Autoregulatory control of $E 2 F 1$ expression in response to positive and negative regulators of cell cycle progression. Genes \& Dev. 8: 1514-1525.

Knoblich, J.A., K. Sauer, L. Jones, H. Richardson, R. Saint, and C.F. Lehner. 1994. Cyclin E controls S phase progression and its down-regulation during Drosophila embryogenesis is required for the arrest of cell proliferation. Cell 77: 107-120.

Kowalik, T.F., J. DeGregori, J.K. Schwarz, and J.R. Nevins. 1995. E2Fl overexpression in quiescent fibroblasts leads to induction of cellular DNA synthesis and apoptosis. I. Virol. 69: 249l-2500.

La Thangue, N.B. 1994. DRTFl/E2F: An expanding family of heterodimeric transcription factors implicated in cell-cycle control. Trends Biochem. Sci. 19: 108-114.

Lowe, S.W. and H.E. Ruley. 1993. Stabilization of the p53 tumor suppressor is induced by adenovirus $5 \mathrm{ElA}$ and accompanies apoptosis. Genes \& Dev. 7: 535-545.

Nevins, J.R. 1992. E2F: A link between the Rb tumor suppressor protein and viral oncoproteins. Science 258: 424-429.

. 1994. Cell cycle targets of the DNA tumor viruses. Curr. Opin. Genet. Dev. 4: 130-134.

Ohtani, K. and J.R. Nevins. 1994. Functional properties of a Drosophila homolog of the E2Fl transcription factor. Mol. Cell. Biol. 14: 1603-1612.

Ohtani, K., J. DeGregori, and J.R. Nevins. 1995. Regulation of the cyclin E gene by the E2Fl transcription factor. Proc. Natl. Acad. Sci. 92: 12146-12150.

Postlethwait, J.H. 1978. Clonal analysis of Drosophila cuticular patterns. In The genetics and biology of Drosophila, Vol. 2c (ed. M. Ashburner and T.R.F. Wright), pp. 359-442. Aca- 
demic Press, New York, NY.

Qin, X.-Q., D.M. Livingston, W.G. Kaelin, and P.D. Adams. 1994. Deregulated transcription factor E2F-1 expression leads to S-phase entry and p53-mediated apoptosis. Proc. Natl. Acad. Sci. 91: 10918-10922.

Ready, D.F., T.E. Hanson, and S. Benzer. 1976. Development of the Drosophila retina, a neurocrystalline lattice. Dev. Biol. 53: $217-240$.

Richardson, H., L.V. O'Keefe, T. Marty, and R. Saint. 1995. Ectopic cyclin $\mathrm{E}$ expression induces premature entry into $\mathrm{S}$ phase and disrupts pattern formation in the Drosophila eye imaginal disc. Development 121: 3371-3379.

Sabbatini, P., S.-K. Chiou, L. Rao, and E. White. 1995. Modulation of p53-mediated transcriptional repression and apoptosis by the adenovirus ElB $19 \mathrm{~K}$ protein. Mol. Cell. Biol. 15: 1060-1070.

Shan, B. and W. Lee. 1994. Deregulated expression of E2F-1 induces S-phase entry and leads to apoptosis. Mol. Cell. Biol. 14: 8166-8173.

Sherr, C.J. 1993. Mammalian $G_{1}$ cyclins. Cell 73: 1059-1065.

Spreij, T.E. 1971. Cell death during the development of the imaginal discs of Calliphora erythrocephala. Neth. J. Zool. 21: 221-264.

Stein, R.W., M. Corrigan, P. Yaciuk, J. Whelan, and E. Moran. 1990. Analysis of ElA-mediated growth regulation functions: Binding of the 300-kilodalton cellular product correlates with E1A enhancer repression function and DNA synthesis-inducing activity. J. Virol. 64: 4421-4427.

Tautz, D. and C. Pleifle. 1989. A non-radioactive in situ hybridization method for the localization of specific RNAs in the Drosophila embryo reveals translational control of the segmentation gene hunchback. Chromosoma 98: 81-85.

Tomlinson, A. 1985. The cellular dynamics of pattern formation in the eye of Drosophila. I. Embryol. Exp. Morphol. 89: 313-331.

Tomlinson, A. and D.F. Ready. 1987a. Neuronal differentiation in the Drosophila ommatidium. Dev. Biol. 120: 366-376.

. 1987b. Cell fate in the Drosophila ommatidium. Dev. Biol. 120: 355-376.

Weinberg, R.A. 1995. The retinoblastoma protein and cell cycle control. Cell 81: 323-330.

White, K., M.E. Grether, J.M. Abrams, L. Young, K. Farrell, and H. Steller. 1994. Genetic control of programmed cell death in Drosophila. Science 264: 677-683.

Wilder, E.L. and N. Perrimon. 1995. Dual function of wingless in the Drosophila leg imaginal disc. Development 121: $477-$ 488.

Wolff, T., and D.F. Ready. 1993. Pattern formation in the Drosophila retina. In The Development of Drosophila melanogaster, Vol. II (ed. M. Bate and A. Martinez Arias) pp. 1277 1325. Cold Spring Harbor Laboratory Press, Cold Spring Harbor, NY.

Wu, X. and A.J. Levine. 1994. p53 and E2F-1 cooperate to mediate apoptosis. Proc. Natl. Acad. Sci. 91: 3602-3606. 


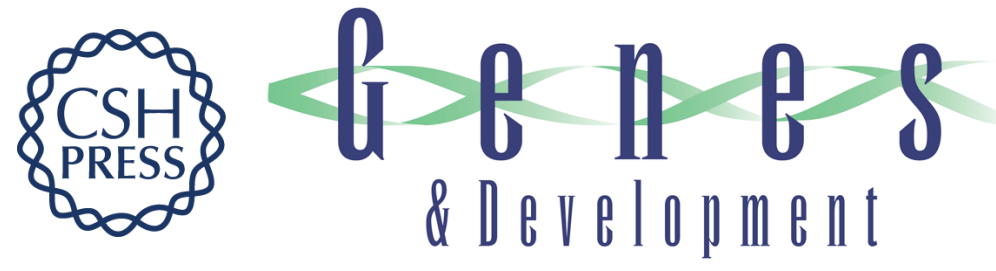

\section{Ectopic E2F expression induces $S$ phase and apoptosis in Drosophila imaginal discs.}

M Asano, J R Nevins and R P Wharton

Genes Dev. 1996, 10:

Access the most recent version at doi:10.1101/gad.10.11.1422

References This article cites 38 articles, 20 of which can be accessed free at:

http://genesdev.cshlp.org/content/10/11/1422.full.html\#ref-list-1

License

Email Alerting

Service

Receive free email alerts when new articles cite this article - sign up in the box at the top right corner of the article or click here.

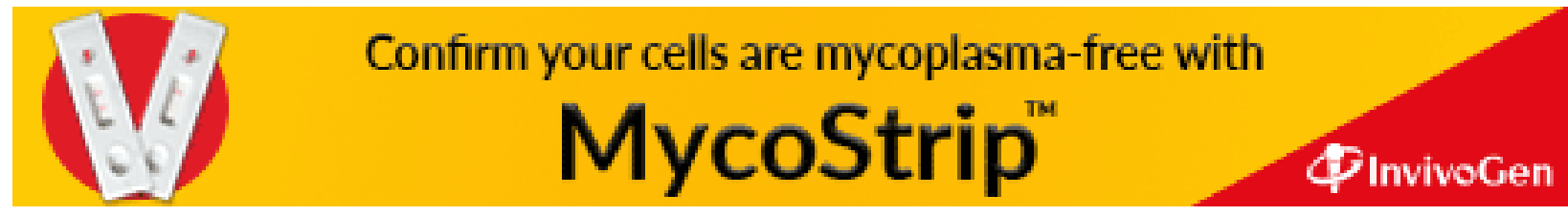

